\title{
ABCA8-mediated efflux of taurocholic acid contributes to gemcitabine insensitivity in human pancreatic cancer via the S1PR2-ERK pathway
}

Chunmei Yang ${ }^{1}$, Hui Yuan ${ }^{1,2,3}$, Jinyang $\mathrm{Gu}^{4}$, Dengfei $\mathrm{Xu}^{1,5}$, Mingwei Wang ${ }^{6}$, Jie Qiao ${ }^{1}, X_{i}$ Yang $^{3,7}$, Jian Zhang ${ }^{3,8}$, Ming Yao ${ }^{1}$, Jianren $\mathrm{Gu}^{1}$, Hong $\mathrm{Tu}^{1}$ and $\mathrm{Yu}$ Gan $\mathbb{1}^{1}$

\begin{abstract}
The development of resistance to anticancer drugs is believed to cause chemotherapy failure in pancreatic cancer (PC). The efflux of anticancer drugs mediated by ATP-binding cassette (ABC) transporters is a widely accepted mechanism for chemoresistance, but for ABCA subfamily members, which are characterized by their ability to transport lipids and cholesterol, its role in chemoresistance remains unknown. Here we found that the expression of $A B C A 8$, a member of ABCA subfamily transporters, was significantly increased in human PC cells after gemcitabine (GEM) treatment, as well as in established GEM-resistant (Gem-R) PC cells. Importantly, ABCA8 knockdown reversed the chemoresistance phenotype of Gem-R cells, whereas ABCA8 overexpression significantly decreased the sensitivity of human PC cells to GEM, both in vitro and in vivo, demonstrating an important role of ABCA8 in regulating chemosensitivity. Moreover, our results showed that treatment with taurocholic acid (TCA), an endogenous substrate of $A B C A 8$, also induced GEM insensitivity in $P C$ cells. We further demonstrated that $A B C A 8$ mediates the efflux of TCA out of PC cells, and that extracellular TCA activates extracellular signal-regulated kinase (ERK) signaling via the sphingosine 1-phosphate receptor 2 (S1PR2), which is responsible for ABCA8-induced GEM ineffectiveness. Together, these findings reveal a novel TCA-related mechanism of ABCA subfamily transportermediated chemoresistance that goes beyond the role of a drug pump and suggest ABCA8 or the TCA-S1RP2-ERK pathway as potential targets for improving the effectiveness of and overcoming the resistance to chemotherapy in PC.
\end{abstract}

\section{Introduction}

Pancreatic cancer (PC) is the fourth leading cause of cancer-related death with a 5 -year relative survival rate of only $9 \%$ and its incidence rate has persistently increased in past decades ${ }^{1,2}$. Although surgery is the current standard of care for resectable PC, chemotherapy is essential for PC

\footnotetext{
Correspondence: Hong Tu (tuhong@shsci.org) or Yu Gan (ganyu@shsci.org) ${ }^{1}$ State Key Laboratory of Oncogenes and Related Genes, Shanghai Cancer Institute, Renji Hospital, Shanghai Jiao Tong University School of Medicine, Shanghai, China

${ }^{2}$ Department of Thoracic Surgery, Cancer Research Center, Fudan University Shanghai Cancer Center, Shanghai, China

Full list of author information is available at the end of the article These authors contributed equally: Chunmei Yang and Hui Yuan Edited by Pier Paolo D'Avino
}

treatment either in an adjuvant setting after surgical resection or in the case of unresectable advanced disease ${ }^{3}$. However, the emergence of chemoresistance limits treatment efficacy and inevitably translates into poor clinical outcomes ${ }^{4,5}$. Therefore, clarifying the molecular mechanisms underlying the chemoresistance of $\mathrm{PC}$ is an urgent need.

ATP-binding cassette (ABC) transporters are known to play a pivotal role in the development of PC chemoresistance due to their ability to pump anticancer drugs out of cancer cells ${ }^{6}$. More than $40 \mathrm{ABC}$ transporters have been identified in human and are divided into 7 subfamilies (ABCA-ABCG) based on amino acid sequence similarities and phylogeny ${ }^{7}$. The most extensively studied

\section{(c) The Author(s) 2021}

\footnotetext{
(c) Open Access This article is licensed under a Creative Commons Attribution 4.0 International License, which permits use, sharing, adaptation, distribution and reproduction cc) in any medium or format, as long as you give appropriate credit to the original author(s) and the source, provide a link to the Creative Commons license, and indicate if changes were made. The images or other third party material in this article are included in the article's Creative Commons license, unless indicated otherwise in a credit line to the material. If material is not included in the article's Creative Commons license and your intended use is not permitted by statutory regulation or exceeds the permitted use, you will need to obtain permission directly from the copyright holder. To view a copy of this license, visit http://creativecommons.org/licenses/by/4.0/.
} 
$A B C$ transporters related to chemoresistance include ABCB1 (also named P-glycoprotein or multidrug resistant gene 1), $\mathrm{ABCC} 1$ (also named multidrug resistance protein 1), and ABCG2 (also named breast cancer resistance protein $)^{8}$. These $A B C$ transporters have been demonstrated to confer PC resistance to gemcitabine $(\mathrm{GEM})^{9-11}$, which is the mainstay chemotherapeutic agent for PC. Several other members of ABCB or ABCC subfamily, such as $A B C B 2^{12}$ and $A B C C 5^{13}$, have also been found to be associated with $\mathrm{PC}$ chemoresistance. However, relatively little is known about the roles of ABCA subfamily transporters in drug resistance.

The ABCA subfamily of transporters consists of 12 members. It was initially recognized for its physiological functions related to transport lipids and cholesterol ${ }^{14}$. For example, ABCA1, ABCA5, and ABCA7 actively export cholesterol and phospholipids ${ }^{15-18}$, and ABCA8 stimulates cholesterol and taurocholic acid (TCA) efflux ${ }^{19}$. A number of previous studies focusing on ABCA transporters reinforced their roles in the maintenance of lipid homeostasis and disorders related to lipid transport ${ }^{14}$. Recently, the effects of $A B C A$ transporters on tumor progression have attracted increasing interest. Regarding their roles in chemotherapy, the expression of several $\mathrm{ABCA}$ transporters, such as $A B C A 1$ and $A B C A 12$, has been reported to be associated with a reduced response to paclitaxel/FEC (5fluorouracil, epirubicin, and cyclophosphamide) neoadjuvant chemotherapy in breast cancer patients ${ }^{20}$. ABCA3 expression defined a class of cancer stem-like cells with high chemoresistance ${ }^{21}$. However, their functional roles in regulating chemo-responsiveness necessitate detailed investigations.

Our recent study found that enriched housing environment-stimulated eustress or positive psycho-social stress increased the sensitivity of mouse PC to GEM and 5-fluorouracil and indicated the involvement of the murine ortholog of human ABCA8 in the regulation of chemosensitivity by eustress ${ }^{22}$. Only a few studies have linked ABCA8 to chemosensitivity in human cancers. A gene expression screen identified a marked increase in ABCA8 expression in the paclitaxel-resistant variants of ovarian cancer cells ${ }^{23}$. In addition, high ABCA8 expression was associated with poor outcome in ovarian can$\operatorname{cer}^{24,25}$. However, the functional roles of ABCA8 and its underlying mechanisms in regulating the chemosensitivity of human cancer remain unknown.

Using both in vitro and in vivo models of human PC, we provide evidence demonstrating that $\mathrm{ABCA} 8$ is involved in the chemoresistance of $\mathrm{PC}$ cells, and that $\mathrm{ABCA} 8$ overexpression leads to a significant decrease in sensitivity to the anticancer drug GEM. Moreover, we further characterized a novel TCA-related mechanism by which ABCA8 induced GEM ineffectiveness beyond acting as a drug pump in PC cells.

\section{Results \\ ABCA8 is upregulated in GEM-treated PC cells and in GEM-resistant (GEM-R) PC cells}

We first examined the changes in ABCA8 expression in response to GEM stimulation in PANC-1 and CFPAC-1 cells, which had relatively low intrinsic expression of $\mathrm{ABCA} 8$ among the five human $\mathrm{PC}$ cell lines we tested (Fig. 1A). As shown in Fig. 1B, C, ABCA8 expression was significantly increased by GEM treatment in a dose-dependent manner at both the mRNA and protein levels. Next, we assessed whether ABCA8 was persistently expressed at a high level in Gem-R cells. Gem-R PC cell lines were established by exposing PANC-1 and CFPAC-1 cells with stepwise increasing concentrations of GEM for 4 months. The half-maximal inhibitory concentration $\left(\mathrm{IC}_{50}\right)$ values of $\mathrm{Gem}-\mathrm{R}$ cells showed that the cells were $\sim 50$-fold more resistant to GEM than parental cells (Fig. 1D). As expected, several well-known chemoresistance-related $\mathrm{ABC}$ transporters, such as ABCG2 and ABCB1, were significantly upregulated in our established Gem-R cells (Supplementary Fig. S1). Notably, the expression of ABCA8 was also substantially increased at both the mRNA and protein levels in these Gem-R cells (Fig. 1E, F), indicating the involvement of $\mathrm{ABCA} 8$ in the acquired chemoresistance of PC. Interestingly, Gem-R cells also exhibited markedly increased migratory and invasive ability (Supplementary Fig. S2a).

\section{ABCA8 knockdown re-sensitizes Gem-R PC cells to GEM in vitro and in vivo}

To determine whether increased ABCA8 expression contributed to acquired chemoresistance, we knocked down ABCA8 in Gem-R cells (Fig. 2A and Supplementary Fig. S3). As shown, the $\mathrm{IC}_{50}$ values for $\mathrm{GEM}$ were substantially reduced by ABCA8 knockdown in Gem-R cells (Fig. 2B). To further confirm the above findings in vivo, nude mice with established subcutaneous xenograft PANC-1 Gem-R shScr or shABCA8 tumors were treated with GEM. As shown in Fig. 2C-E, GEM treatment resulted in a slight but significant reduction in shScr tumors (tumor weight: $0.89 \pm 0.31 \mathrm{~g}$ vs. $0.64 \pm 0.09 \mathrm{~g}, P<$ $0.05)$, whereas the GEM-induced tumor reduction was much more profound in shABCA8 tumors (tumor weight: $0.80 \pm 0.26 \mathrm{~g}$ vs. $0.33 \pm 0.13 \mathrm{~g}, \quad P<0.001)$. The tumor inhibition rate of GEM therapy for Gem-R tumors was increased from $28.5 \%$ to $58.1 \%$ by ABCA8 knockdown (Fig. 2F). These results indicated that ABCA8 contributed to acquired chemoresistance, and that the knockdown of ABCA8 could re-sensitize Gem-R PC cells to GEM therapy.

GEM treatment was able to induce the apoptosis of cancer cells, including PC cells ${ }^{26}$. Here we found that ABCA 8 knockdown significantly promoted GEM-induced 


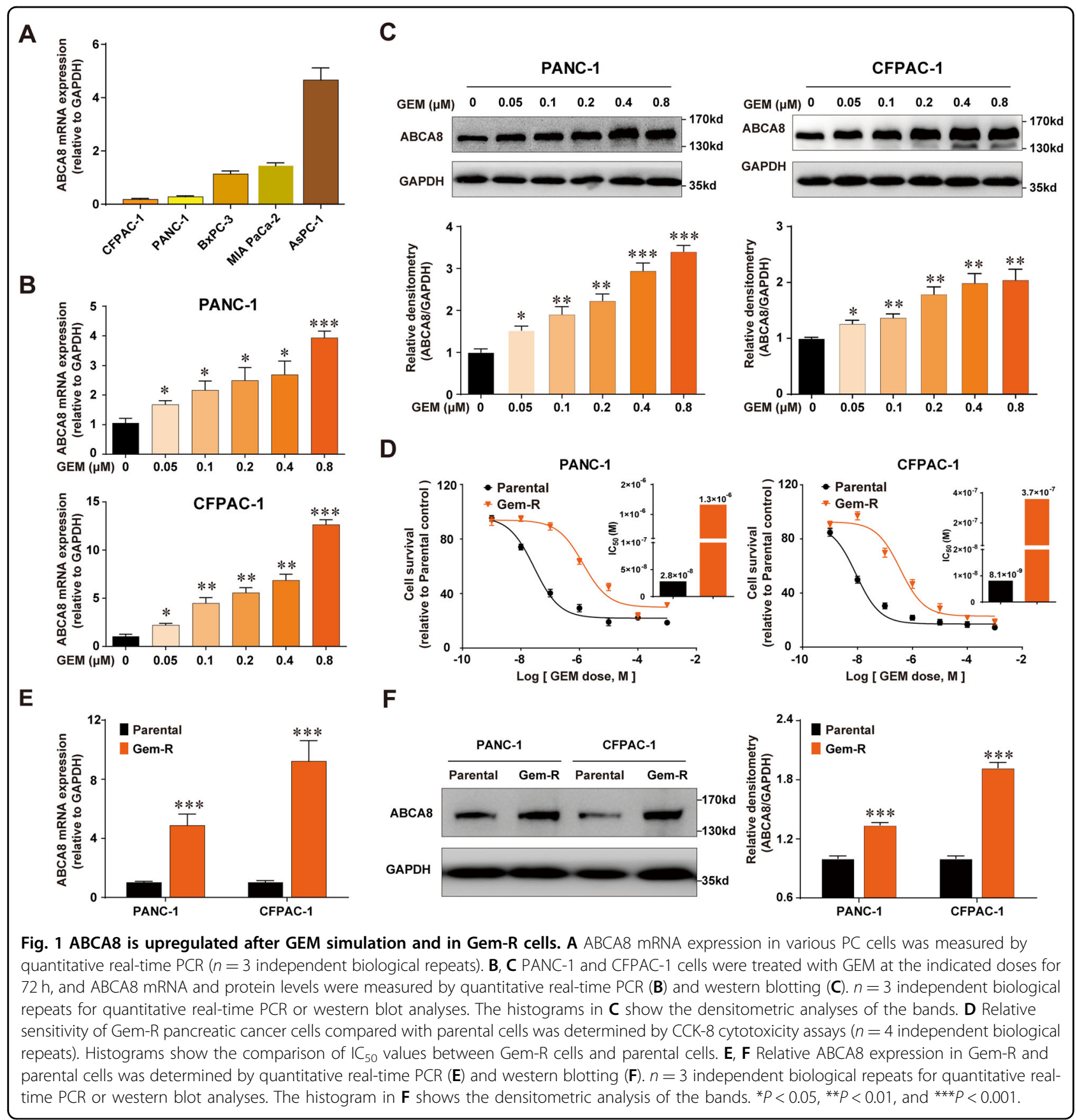

apoptosis in Gem-R cells (Fig. 2G and Supplementary Fig. S4). BCL2 family members play important roles in anticancer drug-induced cancer cell apoptosis. The prosurvival protein BCL2 confered resistance and the pro-apoptosis protein BAX sensitized PC cells to GEMinduced apoptosis ${ }^{27}$. As expected, knockdown of ABCA8 upregulated BAX and downregulated BCL2 in Gem-R cells (Fig. 2A). In addition, ABCA8 knockdown also decreased the migratory and invasive ability of Gem-R cells (Supplementary Fig. S2b).

\section{ABCA8 overexpression decreases the sensitivity of PC cells to GEM in vitro and in vivo}

To further confirm the functional role of $\mathrm{ABCA} 8$ in regulating the chemosensitivity of human $\mathrm{PC}$, we then overexpressed ABCA8 in PANC-1 and CFPAC-1 cells. The overexpression of $\mathrm{ABCA} 8$, which was confirmed by western blotting (Fig. 3A), decreased the sensitivity to GEM in vitro. ABCA8-overexpressing PC cells had substantially higher $\mathrm{IC}_{50}$ values than control cells (Fig. 3B). ABCA8 overexpression also reduced the 5-FU 


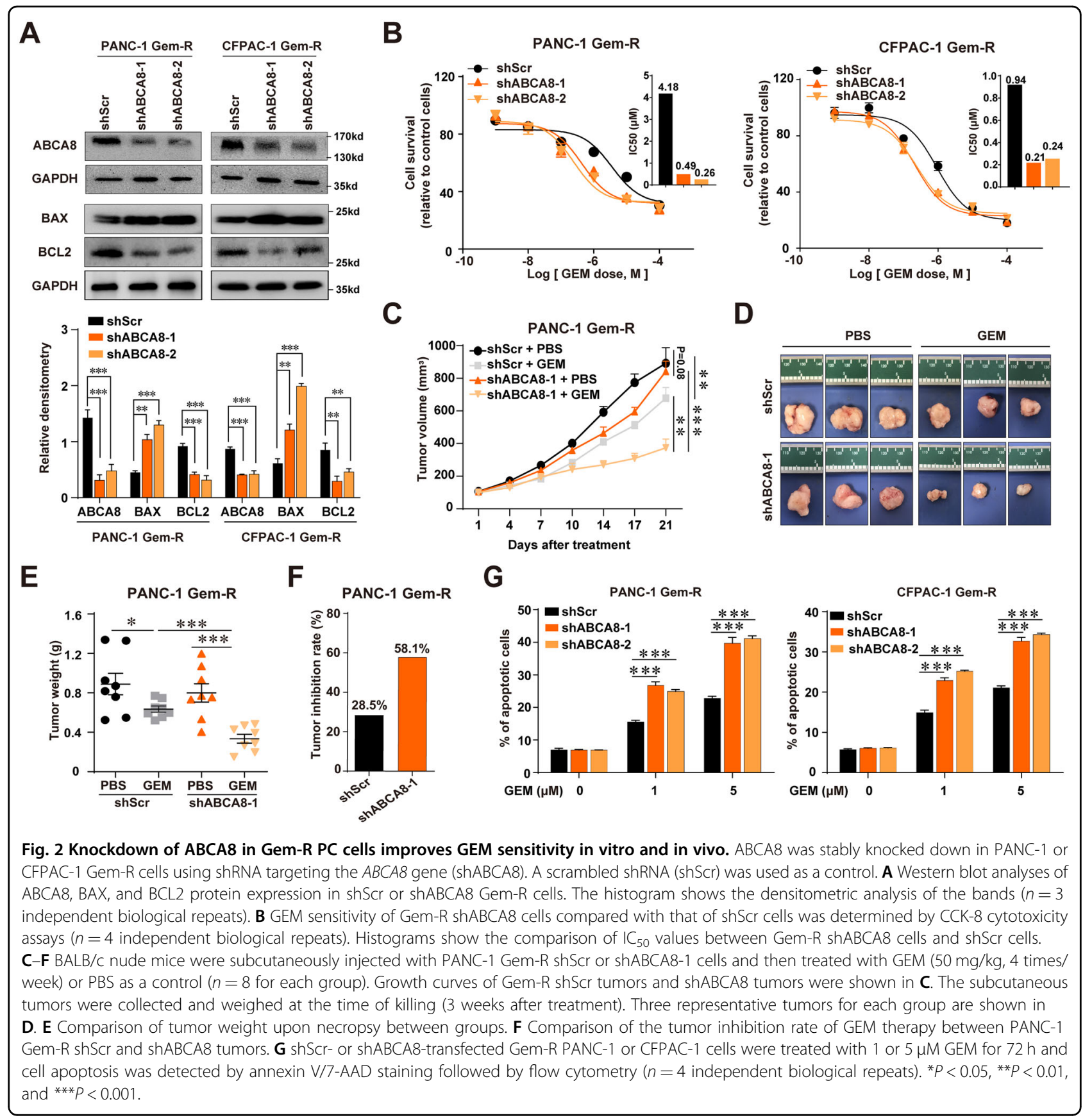

sensitivity of PC cells (Supplementary Fig. S5). However, it failed to significantly reduce the chemosensitivity of the normal human pancreatic duct epithelial hTERT-HPNE cells (Supplementary Fig. S6). We then evaluated the therapeutic efficacy of GEM treatment on ABCA8-overexpressing tumors in nude mice. As shown in Fig. 3C-E, whereas GEM treatment exhibited a profound inhibitory effect on the growth of control tumors (tumor weight: $0.30 \pm 0.07 \mathrm{~g}$ vs. $0.07 \pm 0.05 \mathrm{~g}$, $P<0.001$ ), it failed to achieve comparable therapeutic efficacy for ABCA8-overexpressing tumors (tumor weight: $0.36 \pm 0.12 \mathrm{~g}$ vs. $0.20 \pm 0.07 \mathrm{~g}, P<0.05)$. The tumor inhibition rate was decreased from $73.3 \%$ in control tumors to $45.5 \%$ in ABCA8-overexpressing tumors (Fig. 3F), suggesting that increased ABCA8 expression reduced the sensitivity of human PC cells to GEM therapy.

Meanwhile, ABCA8 overexpression substantially attenuated GEM-induced apoptosis of PC cells (Fig. 3G). Consistently, ABCA8-overexpressing cells exhibited decreased BAX expression and increased BCL2 expression compared with control cells (Fig. 3A). In addition, ABCA8 


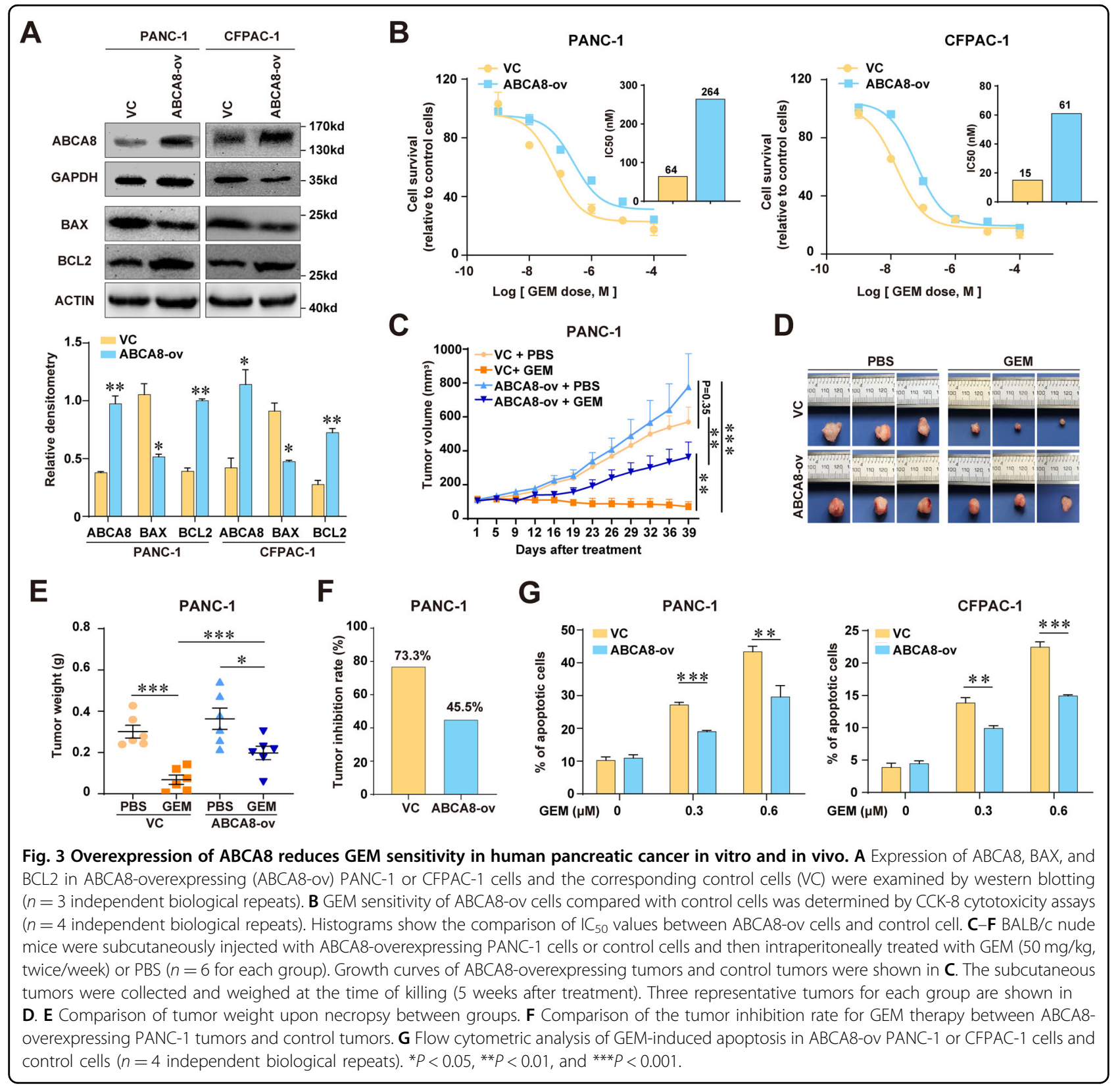

overexpression significantly enhanced the migration and invasion of PC cells (Supplementary Fig. S2c).

\section{$A B C A 8$ induces GEM insensitivity and regulates $B C L 2 / B A X$ balance via ERK signaling in PC cells}

The activation of extracellular signal-regulated kinase (ERK) signaling activation has been involved in the drug resistance of PC cells. Unsurprisingly, increased ERK phosphorylation was observed in our Gem-R cells (Fig. 5A). Interestingly, the results from Gene Ontology enrichment analysis of The Cancer Genome Atlas (TCGA) data showed that high $\mathrm{ABCA} 8$ expression in $\mathrm{PC}$ was significantly associated with "positive regulation of ERK1 and ERK2 cascade"
(Supplementary Fig. S7), indicating that ERK signaling also contributed to the ABCA8-mediated chemoresistance. This was supported by our observation that ABCA 8 knockdown substantially decreased the phosphorylation levels of ERK in Gem-R PC cells (Fig. 4B). In constrast, ABCA8 overexpression increased the ERK phosphorylation (Fig. 4C). Blockade of ERK signaling by its specific ERK inhibitor SCH772984 abrogated ABCA8-induced chemotherapy insensitivity, as evidenced by the comparable GEM-induced cytotoxicity in ABCA8-overexpressing cells to that in control cells in the presence of the ERK-specific inhibitor SCH772984 (Fig. 4D). Furthermore, SCH772984 also reversed the inhibitory effect of $\mathrm{ABCA} 8$ overexpression on 


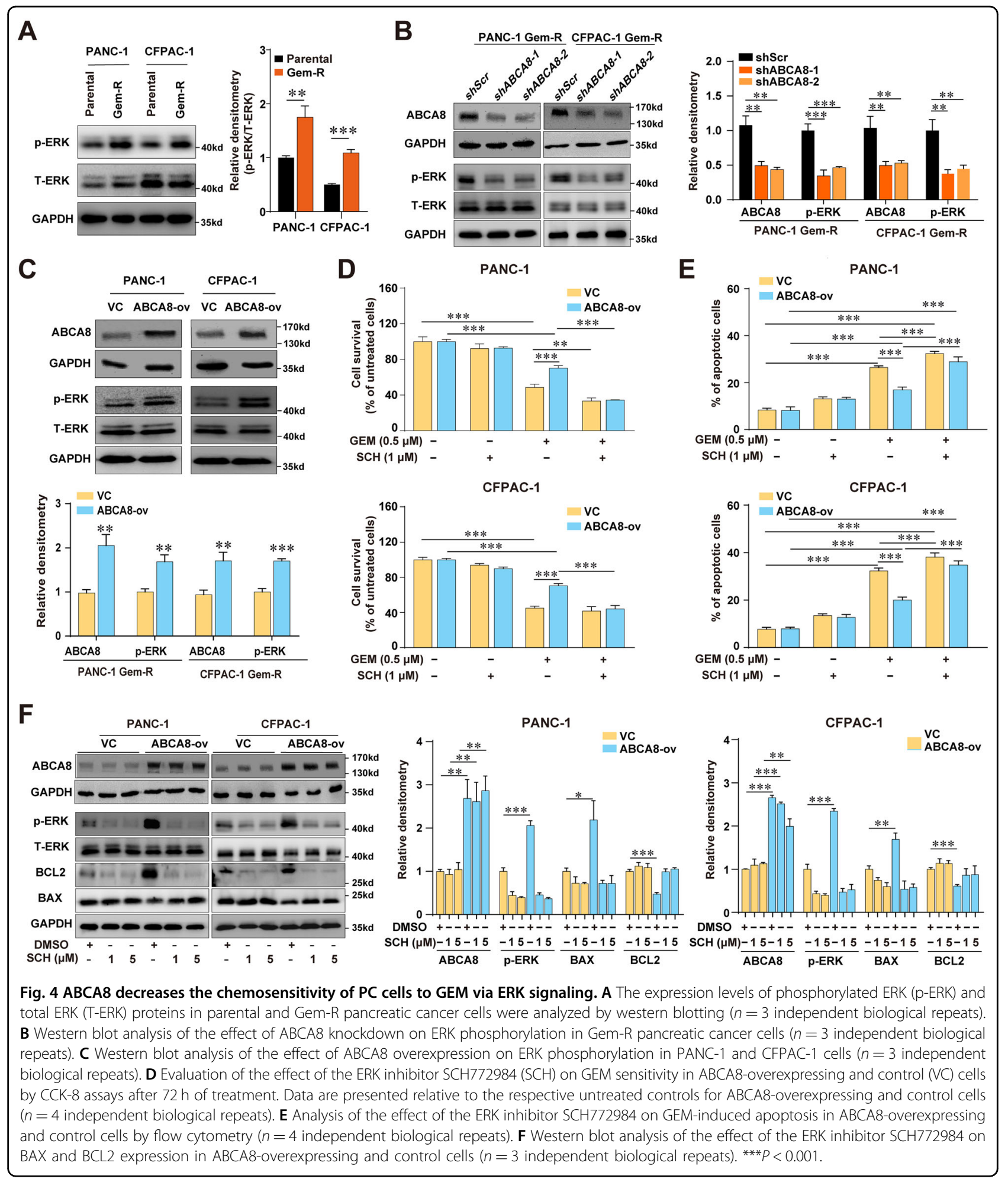

GEM-induced apoptosis of PANC-1 or CFPAC-1 cells (Fig. $4 \mathrm{E}$ ) and abolished the expression changes of BAX and BCL2 induced by $\mathrm{ABCA} 8$ overexpression (Fig. 4F). These results suggested that ABCA8 mediated the GEM insensitivity of PC cells by activating the ERK pathway.
ABCA8 stimulates the efflux of TCA that contributes the GEM resistance in $P C$ cells

ABCA8 has been shown to act as a sinusoidal efflux transporter for $\mathrm{TCA}^{19}$. TCA is a conjugated primary bile acid (BA). Although BAs are mainly biosynthesized in the 


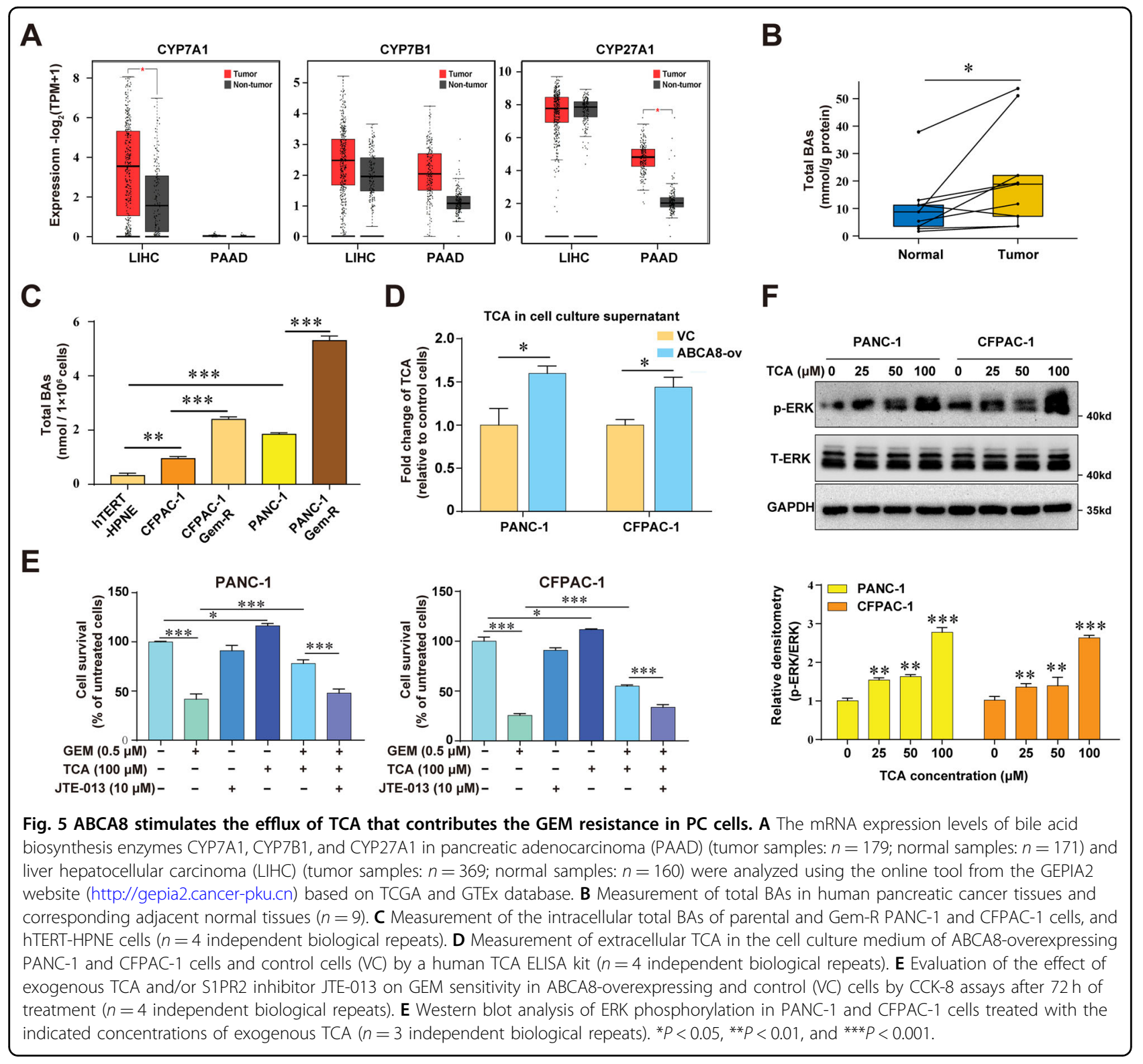

liver, they can also be detected in extrahepatic tissues ${ }^{28}$. Interestingly, the analyses of TCGA and GTEx data showed that PC tissues expressed comparable mRNA levels of CYP27A1 and CYP7B1, two rate-limiting enzymes in the alternative (acidic) pathway of BA biosynthesis, as liver tissues (Fig. 5A). To further determine whether BAs also exist in pancreatic tissue, we measured the total BAs in nine paired human PC tissues and adjacent normal tissues. As shown in Fig. 5B, there were detectable levels of total BAs in human pancreatic tissues and the BA concentration in tumor tissue was significantly higher than that in adjacent normal tissues. In addition, there were significantly higher levels of intracellular BAs in PC cells than in normal pancreatic ductal cells (Fig. 5C). The intracellular BA levels were further increased in Gem-R cells compared with parental cells (Fig. 5C), suggesting a possible role of BAs in regulating the chemosensitivity of PC.

As TCA, one of the predominant conjugate forms of BAs in humans ${ }^{29}$, has been demonstrated as an endogenous substrate of $\mathrm{ABCA} 8^{19}$ and there were significantly higher levels of extracellular TCA in the media of ABCA8-overexpressing cells (Fig. 5D), we further treated PC cells with TCA to determine its functional effect. Extracellular TCA stimulation significantly decreased the sensitivity of both PANC-1 and CFPAC-1 cells to GEM (Fig. 5E). In addition, TCA treatment also led to increased phosphorylation of ERK (Fig. 5F), suggesting that extracellular TCA is involved in regulating the chemosensitivity of PC cells. Further, TCA treatment also led to 


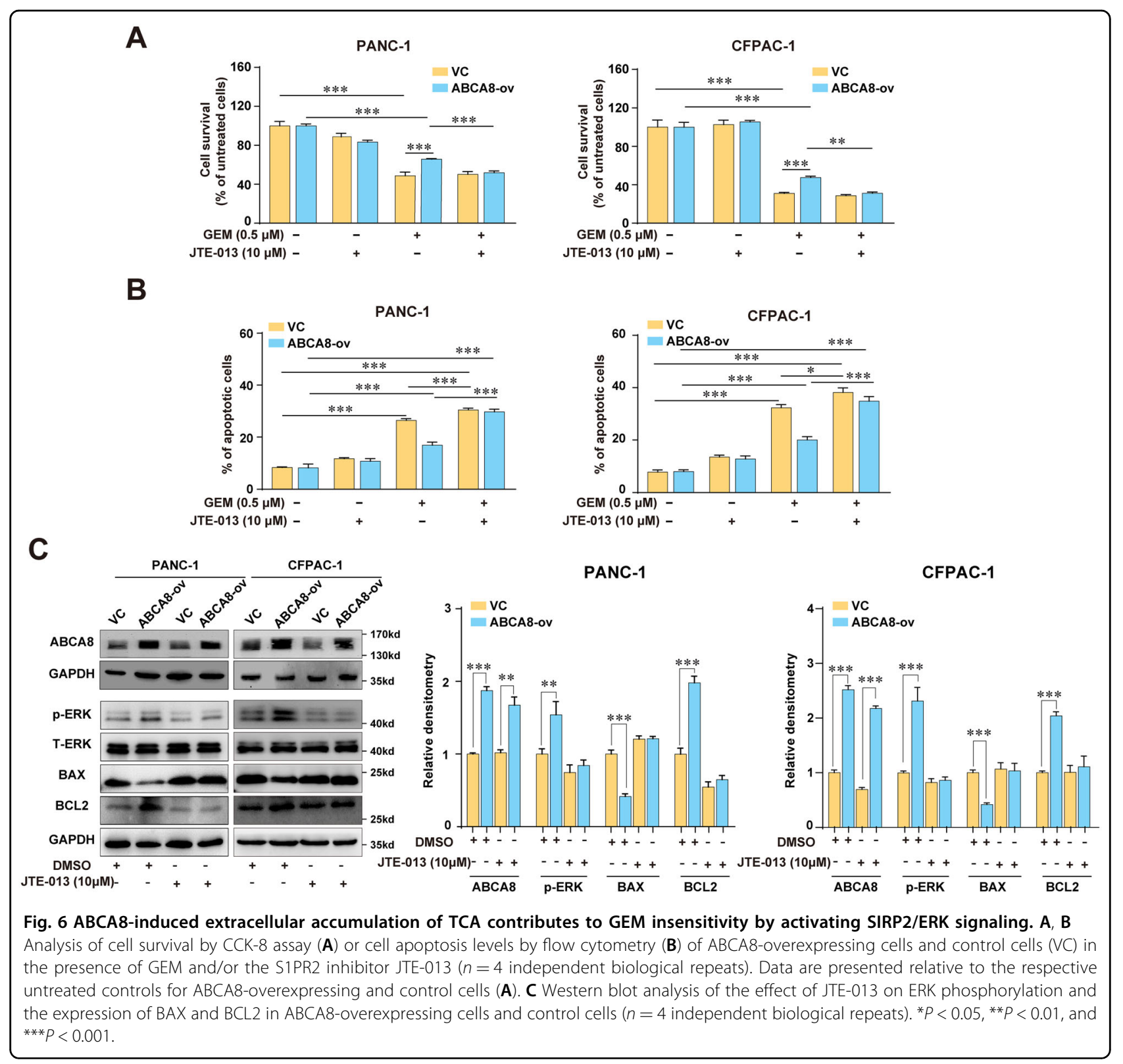

significant enhancement of migratory and invasive properties of PC cells (Supplementary Fig. S2d).

\section{ABCA8-induced extracellular accumulation of TCA decreases GEM sensitivity though S1PR2/ERK pathway}

It has been shown that TCA is a potent signaling agent in the extracellular environment and could act at sphingosine 1-phosphate receptor 2 (S1PR2), a G-proteincoupled receptor, to activate the intracellular signaling pathway $^{30}$. Treatment with the S1PR2-specific inhibitor JTE-013 diminished TCA-induced GEM insensitivity (Fig. 5E), suggesting the involvement of S1PR2 in mediating TCA function in PC cells. Importantly, ABCA8 overexpression failed to decrease the sensitivity to GEM and protect PANC-1 or CFPAC-1 cells against GEMinduced apoptosis in the presense of JTE-013 (Fig. 6A, B). Moreover, blockade of S1PR2 by JTE-013 eliminated the increased phosphorylation of ERK, as well as the expression changes of $\mathrm{BAX}$ and $\mathrm{BCL} 2$, induced by $\mathrm{ABCA} 8$ overexpression (Fig. 6C), suggesting the activation of S1PR2 by TCA as a molecular event upstream of the ERK cascade in ABCA8-overexpressing cells. Together, these results indicated that $\mathrm{ABCA} 8$ induces GEM insensitivity of PC cells by pumping TCA out of cells and then activating the S1PR2-ERK axis. Furthermore, inhibition of S1PR2 with JTE-013 also abolished the enhanced migratory and invasive capability induced by $\mathrm{ABCA} 8$ overexpression or TCA treatment (Supplementary Fig S2c, d). 


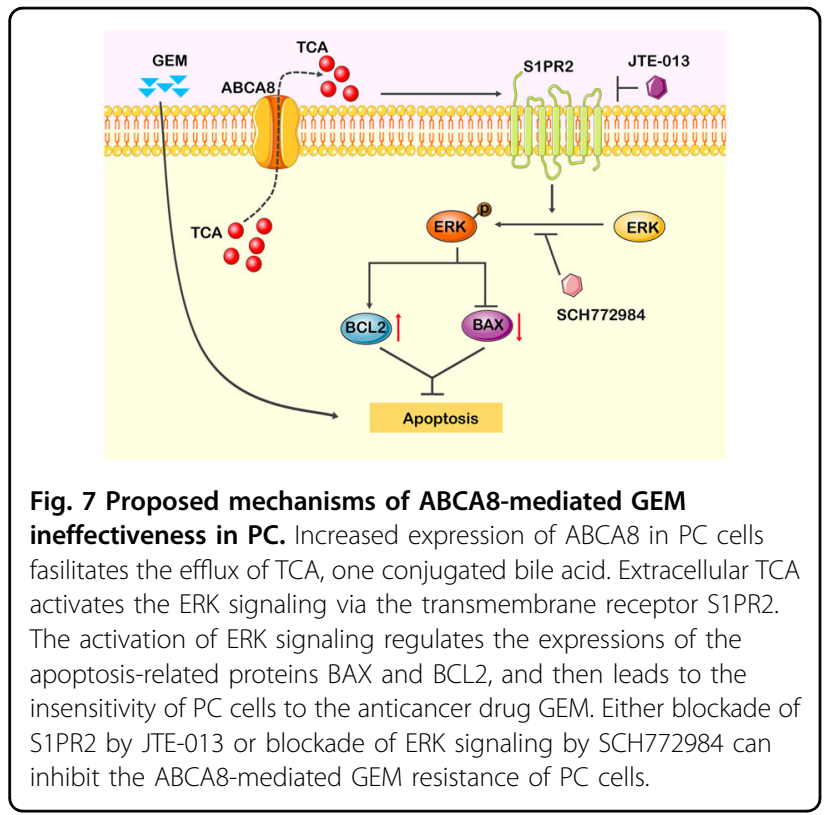

\section{Discussion}

Recently, the roles of ABCA subfamily transporters in cancers have attracted increasing interest ${ }^{14}$. Regarding ABCA8, the increased expression of this ABCA subfamily transporter has been linked to poor prognosis of cancer patients ${ }^{24,25}$. However, its functional role remains unclear. Here, we highlighted the functional importance of $A B C A 8$ in mediating drug resistance of human PC cells based on a combination of both in vitro and in vivo evidence. Although further clinical research is needed, our results suggest $\mathrm{ABCA} 8$ as a potential target for overcoming resistance and improving chemotherapy effectiveness in PC. ABC transporters usually confer chemoresistance due to their ability to efflux anticancer drugs from cancer cells $^{31,32}$. Rather than their role as a drug efflux pump, the current study further presents a novel mechanism of $A B C$ transporter-mediated chemoresistance in $\mathrm{PC}$ in which the ABCA subfamily member ABCA8-mediated efflux of TCA contributes to GEM ineffectiveness by activating S1PR2/ERK signaling (Fig. 7).

TCA is a taurine-conjugated form of primary BA. BA is considered to be synthesized mainly in the liver due to the extremely low expression level of the rate-limiting BA biosynthesis enzyme CYP7A1 in extrahepatic tissues ${ }^{33}$. However, BA can also be synthesized via the alternative (acidic) pathway with the rate-limiting enzymes CYP27A1 and CYP7B1, whose expressions are not restricted to the liver $^{28}$. Based on the analyses of TCGA and GTEx data, pancreatic tissues were found to express comparable mRNA levels of CYP27A1 and CYP7B1 as liver tissues despite the extremely low level of CYP7A1. Moreover, we found that there were detectable levels of BAs in pancreatic tissue, as well as in cell lines derived from human normal pancreatic duct or PC. The levels of total BA or CYP27A1 expression were even higher in PC compared to normal pancreatic cells or tissues. Therefore, it is reasonable to believe the existence of BA biosynthesis via the acidic pathway in pancreatic tissue, particularly in PC.

Increasing attention has been paid to the roles of BAs in cancer development and progression. As a predominant BA, TCA was found to promote the invasive growth of cholangiocarcinoma and esophageal adenocarcinoma ${ }^{34,35}$. However, the effect of TCA on the response to anticancer drugs has not yet been studied. Here we showed that extracellular TCA decreased the sensitivity of PC cells to GEM. This observation, together with the fact that ABCA8 facilitates TCA efflux, suggested that ABCA8mediated extracellular accumulation of TCA contributes to the decreased GEM sensitivity induced by ABCA8. We also noted that, in the normal pancreatic duct epithelial hTERT-HPNE cells that had the markedly lower level of $\mathrm{BA}$ biosynthesis than PC cells, ABCA8 overexpression failed to decrease their GEM sensitivity. This observation further strengthened the importance of TCA efflux in ABCA8-induced chemoresistance. It was reported before that S1PR2, which is a member of the sphingosine 1-phosphate G-protein-coupled receptor family ${ }^{36}$, is a transmembrane receptor of extracellular $\mathrm{TCA}^{37,38}$. After blockade of S1PR2, we observed a reversal of chemoresistance either in ABCA8-overexpressing cells or in TCAtreated cells. These findings suggested the involvement of TCA and its downstream S1PR2-mediated signaling in the regulation of chemosensitivity and extended our understanding of the role of BAs in cancers.

Our results indicated that TCA-S1PR2-ERK signalingmediated apoptotic resistance played a role in ABCA8induced GEM ineffectiveness. Conjugated BAs have been reported to activate the ERK signaling pathway via S1PR2 in rodent hepatocytes ${ }^{30}$ and human cholangiocarcinoma cells $^{38}$. Consistent with those reported, our study also linked TCA-mediated activation of S1PR2 to ERK signaling in PC cells, and demonstrated that SIPR2-ERK is a critical intracellular signal that mediates ABCA8-induced GEM ineffectiveness. The activation of ERK was shown to confer resistance to GEM-induced apoptosis by regulating the expression of the apoptosis-related proteins BCL2 and $\mathrm{BAX}^{27}$. In ABCA8-overexpressing PC cells, the activation of ERK was associated not only with apoptosis resistance but also with the expression changes of BCL2 and BAX. Notably, ERK signaling is involved in resistance to other anticancer drugs in addition to $\mathrm{GEM}^{39-41}$. In fact, overexpression of ABCA8 in PC cells also reduced their sensitivity to 5-FU, suggesting the involvement of ABCA8-TCA-S1PR2-ERK pathway in the development of multidrug resistance in $\mathrm{PC}$ cells.

An increasing body of evidence indicates that chemotherapy can increase the metastatic potential of cancer 
cells $^{42}$. In particular, GEM treatment has been shown to enhance the cell migration and invasiveness of PC and lung cancer ${ }^{43,44}$. These findings were supported by our observation that Gem-R cells exhibited markedly increased migratory and invasive ability in this study. Interestingly, this study further found that $\mathrm{ABCA} 8$ overexpression could enhance the migration and invasion of PC cells and suggested the contribution of TCA-S1PR2 pathway to $A B C A 8$-induced cell migration and invasion. Given that ABCA 8 was significantly upregulated by GEM, we can reasonably speculate that ABCA8-TCA-S1PR2 axis might serve as an additional mechanism of GEM therapy-induced metastasis in PC, which awaits further studies to confirm.

In conclusion, our study revealed the role of ABCA8mediated TCA efflux in regulating PC cells sensitivity to chemotherapy and characterized the TCA-S1PR2-ERK pathway underlying ABCA8-induced GEM ineffectiveness. These findings suggest that targeting $A B C A 8$ or the TCA-S1PR2-ERK pathway represents new strategies for improving the effectiveness of and overcoming the resistance to chemotherapy in PC.

\section{Materials and methods}

\section{Cell lines and culture}

Human PC cell lines (PANC-1, CFPAC-1, BxPC-3, AsPC-1, MIA PaCa-2) and immortalized normal pancreatic duct epithelial hTERT-HPNE cells were obtained from the American Type Culture Collection (Manassas, VA, USA). Cells were cultured in Dulbecco's modified Eagle's medium (hTERT-HPNE, PANC-1, MIA PaCa-2), RPMI-1640 (CFPAC-1, BxPC-3), or IMDM (AsPC-1) medium supplemented with $10 \%$ fetal bovine serum (Thermo Scientific, Waltham, MA, USA) in a humidified atmosphere of $5 \% \mathrm{CO}_{2}$ at $37^{\circ} \mathrm{C}$. All cell lines were authenticated using standard short tandem repeat testing by Biowing Applied Biotechnology, Co., Ltd (Shanghai, China). For details about the generation of Gem-R cells, ABCA8-overexpressing cells, or ABCA8-knockdown cells, see Supplementary Materials and Methods.

\section{Quantitative real-time PCR}

RNA isolation from GEM-treated or Gem-R PC cells and quantitative real-time PCR analysis of $A B C$ transporter expression were performed according to our previous description ${ }^{45}$. The relative expression of the target genes was normalized to GAPDH. The primer sequences are provided in Supplementary Table S1.

\section{Western blotting}

Western blot analysis was performed according to the procedure described previously ${ }^{45}$. The antibodies used for western blotting are shown in Supplementary Table S2. All western blottings were quantified using ImageJ software and the quantitative results were presented as the relative expression levels of target proteins normalized to the corresponding loading controls or pan-protein levels. The western blotting images shown are representative of three independent experiments.

\section{Cell viability and cell apoptosis assays}

Cell viability was monitored using a CCK-8 (Dojindo, Kumamoto, Japan) and cell apoptosis was analyzed using the PE Annexin V Apoptosis Detection Kit (BioLegend, San Diego, CA, USA) according to the corresponding manufacturer's protocols. For details about the procedure of these assays, see Supplementary Materials and Methods.

\section{Animal experiments}

Male athymic BALB/c nude mice were used in this study and maintained under specific pathogen-free conditions. Four-week-old nude mice were subcutaneously inoculated with cancer cells $\left(5 \times 10^{6}\right.$ cells/mouse $)$ and the tumor size was monitored. When the tumor volume reached $100 \mathrm{~mm}^{3}$, the nude mice were randomly assigned to groups and treated with vehicle or GEM $(50 \mathrm{mg} / \mathrm{kg}$, 4 times/week for $\mathrm{ABCA} 8$ knockdown experiments and $50 \mathrm{mg} / \mathrm{kg}, 2$ times/week for ABCA8 overexpression experiments). Four or 8 weeks after tumor inoculation, mice were killed and the tumors were excised and weighed. The tumor inhibition rate was calculated using the following formula: inhibition rate $(\%)=($ mean tumor weight of phosphate-buffered saline (PBS)-treated group - mean tumor weight of the GEM-treated group)/mean tumor weight of PBS-treated group. All animal experiments were conducted double-blind and performed according to the protocols approved by the Medical Experimental Animal Care Commission at the Shanghai Cancer Institute.

\section{$B A$ and TCA measurements}

For BA measurement in PC tissues, nine paired PC and adjacent normal tissues were collected from patients pathologically diagnosed with PC in the Third Hospital Affiliated to Nantong University from October 2019 to January 2020. Informed consent was obtained from each patient prior to sample collection. This study was approved by the Ethics Committee of the Third Hospital Affiliated to Nantong University.

The concentration of total BAs was determined by the Total Bile Acids Assay Kit (BioVision, Mountain View, CA, USA) and the concentration of TCA was determined by using the Human Taurocholic Acid Detection ELISA kit (Enzyme-linked Biotechnology, Shanghai, China) according to the corresponding manufacturer's guidelines. For details about the procedure of these assays, see Supplementary Materials and Methods. 


\section{Statistical analysis}

Quantitative data are represented as the mean \pm SEM of at least three independent repeated experiments. GraphPad Prism 7.0 software was used to perform statistical analysis. The significance of differences was assessed by Student's $t$-test for single comparisons or by analysis of variance with the Tukey's test for multiple comparisons. $P$-values $<0.05$ were considered statistically significant.

\section{Acknowledgements}

This work was supported by grants from the National Natural Science Foundation of China (81872505, 81572312 to H.T., 81502601 to H.Y., 81571618 to Y.G.) and Chinese State Key Laboratory of Oncogenes and Related Genes (97-11-30 and ZZ2004RCPY to Y.G.).

\begin{abstract}
Author details
'State Key Laboratory of Oncogenes and Related Genes, Shanghai Cancer Institute, Renji Hospital, Shanghai Jiao Tong University School of Medicine, Shanghai, China. 'Department of Thoracic Surgery, Cancer Research Center, Fudan University Shanghai Cancer Center, Shanghai, China. ${ }^{3}$ Department of Oncology, Shanghai Medical College, Fudan University, Shanghai, China. ${ }^{4}$ Department of Transplantation, Xinhua Hospital, Shanghai Jiao Tong University School of Medicine, Shanghai, China. ${ }^{5}$ Department of Oncology, Henan Province People's Hospital, Zhengzhou, Henan Province, China. ${ }^{6}$ Department of Radiation Oncology, The Third Hospital Affiliated to Nantong University, Nantong, Jiangsu Province, China. ${ }^{7}$ Department of Radiation Oncology, Fudan University Shanghai Cancer Center, Shanghai, China. ${ }^{8}$ Department of Medical Oncology, Fudan University Shanghai Cancer Center, Shanghai, China
\end{abstract}

\section{Author contributions}

YG and HT conceived the study and designed the project. CY and HY carried out the experiments and analyzed the data. YG and CY wrote the manuscript. Jingyang Gu, DX, MW, JQ, XY, and JZ performed some experiments and helped process the experimental data. HT, MY, and Jianren Gu aided in interpreting the results and contributed to the revision of the manuscript. All authors approved the final version of the manuscript.

\section{Conflict of interest}

The authors declare that they have no conflict of interest.

\section{Publisher's note}

Springer Nature remains neutral with regard to jurisdictional claims in published maps and institutional affiliations.

The online version of this article (https://doi.org/10.1038/s41420-020-00390-z) contains Supplementary Materials, which is available to authorized users.

Received: 28 July 2020 Revised: 9 November 2020 Accepted: 9 December 2020

Published online: 11 January 2021

\section{References}

1. Siegel, R. L., Miller, K. D. \& Jemal, A. Cancer statistics, 2019. CA Cancer J. Clin. 69, 7-34 (2019).

2. Borazanci, E. et al. Pancreatic cancer: "a riddle wrapped in a mystery inside an enigma". Clin. Cancer Res. 23, 1629-1637 (2017).

3. Li, Q. et al. NF-kappaB in pancreatic cancer: its key role in chemoresistance. Cancer Lett. 421, 127-134 (2018).

4. Biffi, G. et al. IL1-induced JAK/STAT signaling is antagonized by TGFbeta to shape CAF heterogeneity in pancreatic ductal adenocarcinoma. Cancer Discov. 9, 282-301 (2019).

5. Shukla, S. K. et al. MUC1 and HIF-1alpha signaling crosstalk induces anabolic glucose metabolism to impart gemcitabine resistance to pancreatic cancer. Cancer Cell 32, 71-87 e77 (2017).
6. Adamska, A. \& Falasca, M. ATP-binding cassette transporters in progression and clinical outcome of pancreatic cancer: what is the way forward? World J. Gastroenterol. 24, 3222-3238 (2018).

7. Dean, M. \& Annilo, T. Evolution of the ATP-binding cassette (ABC) transporter superfamily in vertebrates. Annu. Rev. Genomics Hum. Genet. 6, 123-142 (2005).

8. Li, W. et al. Overcoming $A B C$ transporter-mediated multidrug resistance: molecular mechanisms and novel therapeutic drug strategies. Drug Resist. Updat. 27, 14-29 (2016).

9. Lu, Y. et al. HNF1A inhibition induces the resistance of pancreatic cancer cells to gemcitabine by targeting ABCB1. EBioMedicine 44, 403-418 (2019).

10. Qian, Y., et al. Coix seed extract enhances the anti-pancreatic cancer efficacy of gemcitabine through regulating $A B C B 1$ - and ABCG2-mediated drug efflux: a bioluminescent pharmacokinetic and pharmacodynamic study. Int. J. Mol. Sci. 20, 5250 (2019)

11. He, X. et al. Hypoxia regulates ABCG2 activity through the activivation of ERK1/ 2/HIF-1alpha and contributes to chemoresistance in pancreatic cancer cells. Cancer Biol. Ther. 17, 188-198 (2016).

12. $\mathrm{Xu}, \mathrm{M}$. et al. ABCB2 (TAP1) as the downstream target of $\mathrm{SHH}$ signaling enhances pancreatic ductal adenocarcinoma drug resistance. Cancer Lett. 333, 152-158 (2013)

13. Nambaru, P. K. et al. Drug efflux transporter multidrug resistance-associated protein 5 affects sensitivity of pancreatic cancer cell lines to the nucleoside anticancer drug 5-fluorouracil. Drug Metab. Dispos. 39, 132-139 (2011).

14. Pasello, M., Giudice, A. M. \& Scotlandi, K. The ABC subfamily A transporters: multifaceted players with incipient potentialities in cancer. Semin. Cancer Biol. 60, 57-71 (2020)

15. Du, X. M. et al. HDL particle size is a critical determinant of ABCA1-mediated macrophage cellular cholesterol export. Circ. Res. 116, 1133-1142 (2015).

16. Fu, Y., Hsiao, J. H., Paxinos, G., Halliday, G. M. \& Kim, W. S. ABCA5 regulates amyloid-beta peptide production and is associated with Alzheimer's disease neuropathology. J. Alzheimers Dis. 43, 857-869 (2015).

17. Vedhachalam, $C$. et al. Mechanism of ATP-binding cassette transporter A1mediated cellular lipid efflux to apolipoprotein A-I and formation of high density lipoprotein particles. J. Biol. Chem. 282, 25123-25130 (2007).

18. Ikeda, Y. et al. Posttranscriptional regulation of human ABCA7 and its function for the apoA-l-dependent lipid release. Biochem. Biophys. Res. Commun. 311, 313-318 (2003).

19. Sasaki, K. et al. ATP-binding cassette transporter A subfamily 8 is a sinusoidal efflux transporter for cholesterol and taurocholate in mouse and human liver. Mol. Pharm. 15, 343-355 (2018).

20. Park, S. et al. Gene expression profiling of ATP-binding cassette (ABC) transporters as a predictor of the pathologic response to neoadjuvant chemotherapy in breast cancer patients. Breast Cancer Res. Treat. 99, 9-17 (2006).

21. Hirschmann-Jax, C. et al. A distinct "side population" of cells with high drug efflux capacity in human tumor cells. Proc. Natl Acad. Sci. USA 101, 14228-14233 (2004).

22. Wu, Y. et al. Enriched environment housing enhances the sensitivity of mouse pancreatic cancer to chemotherapeutic agents. Biochem. Biophys. Res. Commun. 473, 593-599 (2016).

23. Januchowski, R. et al. Drug transporter expression profiling in chemoresistant variants of the A2780 ovarian cancer cell line. Biomed. Pharmacother. 68 447-453 (2014).

24. Liu, X. et al. Discovery of microarray-identified genes associated with ovarian cancer progression. Int. J. Oncol. 46, 2467-2478 (2015).

25. Hedditch, E. L., et al. ABCA transporter gene expression and poor outcome in epithelial ovarian cancer. J. Natl Cancer Inst. 106, dju149 (2014).

26. Ma, J., Hui, P. Meng, W., Wang, N. \& Xiang, S. Ku70 inhibits gemcitabineinduced DNA damage and pancreatic cancer cell apoptosis. Biochem. Biophys. Res. Commun. 484, 746-752 (2017).

27. Wang, $M$. et al. pERK1/2 silencing sensitizes pancreatic cancer BXPC-3 cell to gemcitabine-induced apoptosis via regulating $\mathrm{Bax}$ and $\mathrm{BCl}-2$ expression. World J. Surg. Oncol. 13, 66 (2015).

28. Hu, M. M. et al. Virus-induced accumulation of intracellular bile acids activates the TGR5-beta-arrestin-SRC axis to enable innate antiviral immunity. Cell Res. 29, 193-205 (2019).

29. Mathavan, S., Mikov, M., Golocorbin-Kon, S. \& Al-Salami, H. Diabetes development increased concentrations of the conjugated bile acid, taurocholic acid in serum, while treatment with microencapsulated-taurocholic acid exerted no hypoglycaemic effects. Eur. J. Pharm. Sci. 106, 1-9 (2017).

30. Studer, E. et al. Conjugated bile acids activate the sphingosine-1-phosphate receptor 2 in primary rodent hepatocytes. Hepatology 55, 267-276 (2012). 
31. Fletcher, J. I., Williams, R. T., Henderson, M. J., Norris, M. D. \& Haber, M. ABC transporters as mediators of drug resistance and contributors to cancer cell biology. Drug Resist. Updat. 26, 1-9 (2016).

32. Beretta, G. L., Cassinelli, G., Pennati, M., Zuco, V. \& Gatti, L. Overcoming ABC transporter-mediated multidrug resistance: the dual role of tyrosine kinase inhibitors as multitargeting agents. Eur. J. Med. Chem. 142, 271-289 (2017)

33. Russell, D. W. The enzymes, regulation, and genetics of bile acid synthesis. Annu. Rev. Biochem. 72, 137-174 (2003).

34. Liu, R., Li, X., Hylemon, P. B. \& Zhou, H. Conjugated bile acids promote invasive growth of esophageal adenocarcinoma cells and cancer stem cell expansion via sphingosine 1-phosphate receptor 2-mediated Yes-associated protein activation. Am. J. Pathol. 188, 2042-2058 (2018).

35. Amonyingcharoen, S., Suriyo, T., Thiantanawat, A., Watcharasit, P. \& Satayavivad, J. Taurolithocholic acid promotes intrahepatic cholangiocarcinoma cell growth via muscarinic acetylcholine receptor and EGFR/ERK1/2 signaling pathway. Int. J. Oncol. 46, 2317-2326 (2015).

36. Adada, M., Canals, D., Hannun, Y. A. \& Obeid, L. M. Sphingosine-1-phosphate receptor 2. FEBS J. 280, 6354-6366 (2013).

37. Liu, R. et al. Taurocholate induces cyclooxygenase-2 expression via the sphingosine 1-phosphate receptor 2 in a human cholangiocarcinoma cell line. J. Biol. Chem. 290, 30988-31002 (2015).

38. Liu, R. et al. Conjugated bile acids promote cholangiocarcinoma cell invasive growth through activation of sphingosine 1-phosphate receptor 2. Hepatology 60, 908-918 (2014).
39. Li, Y. et al. ERK inhibition effectively overcomes acquired resistance of epidermal growth factor receptor-mutant non-small cell lung cancer cells to osimertinib. Cancer 126, 1339-1350 (2020).

40. Wang, $X$. et al. IL-22 confers EGFR-TKI resistance in NSCLC via the AKT and ERK signaling pathways. Front. Oncol. 9, 1167 (2019).

41. Zhu, L., Ding, R., Yan, H., Zhang, J. \& Lin, Z. ZHX2 drives cell growth and migration via activating MEK/ERK signal and induces Sunitinib resistance by regulating the autophagy in clear cell renal cell carcinoma. Cell Death Dis. 11, 337 (2020).

42. Karagiannis, G. S., Condeelis, J. S. \& Oktay, M. H. Chemotherapy-induced metastasis: mechanisms and translational opportunities. Clin. Exp. Metastasis 35, 269-284 (2018).

43. Ahmmed, B. et al. Rg3 inhibits gemcitabine-induced lung cancer cell invasiveness through ROS-dependent, NF-kappaB- and HIF-1alphamediated downregulation of PTX3. J. Cell Physiol. 234, 10680-10697 (2019).

44. Arora, S. et al. An undesired effect of chemotherapy: gemcitabine promotes pancreatic cancer cell invasiveness through reactive oxygen species-dependent, nuclear factor kappaB- and hypoxia-inducible factor 1alpha-mediated up-regulation of CXCR4. J. Biol. Chem. 288, 21197-21207 (2013).

45. Xu, D. et al. Cadherin 13 inhibits pancreatic cancer progression and epithelialmesenchymal transition by Wnt/beta-catenin signaling. J. Cancer $\mathbf{1 1}$ 2101-2112 (2020). 